\title{
Thailand's experience on radiation safety and quality practice in diagnostic radiology
}

\author{
Napapong Pongnapang, Ph.D. \\ From Department of Radiological Technology, Faculty of Medical Technology, \\ Mahidol University, Bangkok, Thailand. \\ Address correspondence to N.P. (e-mail: napapong.pon@mahidol.ac.th)
}

Received 24 December 2020; accepted 25 December 2020

doi:10.46475/aseanjr.v21i3.101

Keywords: Thailand, Radiology, Radiation safety, Quality.

Radiology services in Thailand started in 1898 by Professor Hans Adamsen, a royal court physician, who brought the first x-ray machine to the country. Thailand's radiology education and training started in 1928 by Professor Pin Muangman, a pioneer radiologist who graduated from Harvard University, USA. At present, Thailand has 1950 radiologists, 200 medical physicists and 5200 radiological technologists practicing in the healthcare system. Radiology services in Thailand started in 1898 by Professor Hans Adamsen, a royal court physician, who brought first x-ray machine to the country. Thailand Radiology education and training started in 1928 by Professor Pin Muangman (Figure 1), a pioneer radiologist who graduated from Harvard University, USA. At present, Thailand has 1950 radiologists, 200 medical physicists and 5200 radiological technologists practicing in the healthcare system. 


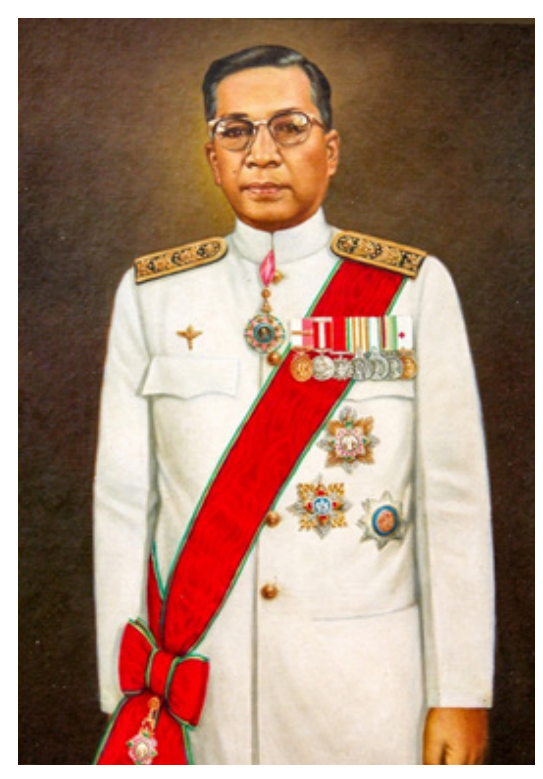

Figure 1. Professor Pin Muangman, M.D., founder of Thailand's Radiology.

A main radiation safety control is under the Nuclear Power Safety Act (updated in 2016). Radiologists and Technologists' professional practices are controlled under a relevant professional law while the medical physicists licensing will be effective by 2021 . An updated regulation stipulates that all radiation emitting machines (in diagnostic radiology and dentistry) are required to be registered at the Ministry of Public Health. Annual safety and quality investigation of $\mathrm{x}$-rays equipment is required to be performed by the physicists.

As a UN member state, Thailand has been actively participating in a number of projects related to quality and safety in diagnostic radiology with the International Atomic Energy Agency (IAEA). Following corresponding requests, usually linked, but not limited to relevant TC projects, the IAEA is responsible for forming the auditing team, giving an international perspective and assuring the principle of independency between the auditors and the audited department. The IAEA clinical audit program (QUAADRIL) was extablished in Thailand in 2017 (Figure 2) with a future plan to adopt the system and transfer it to Thai-QUAADRIL. 


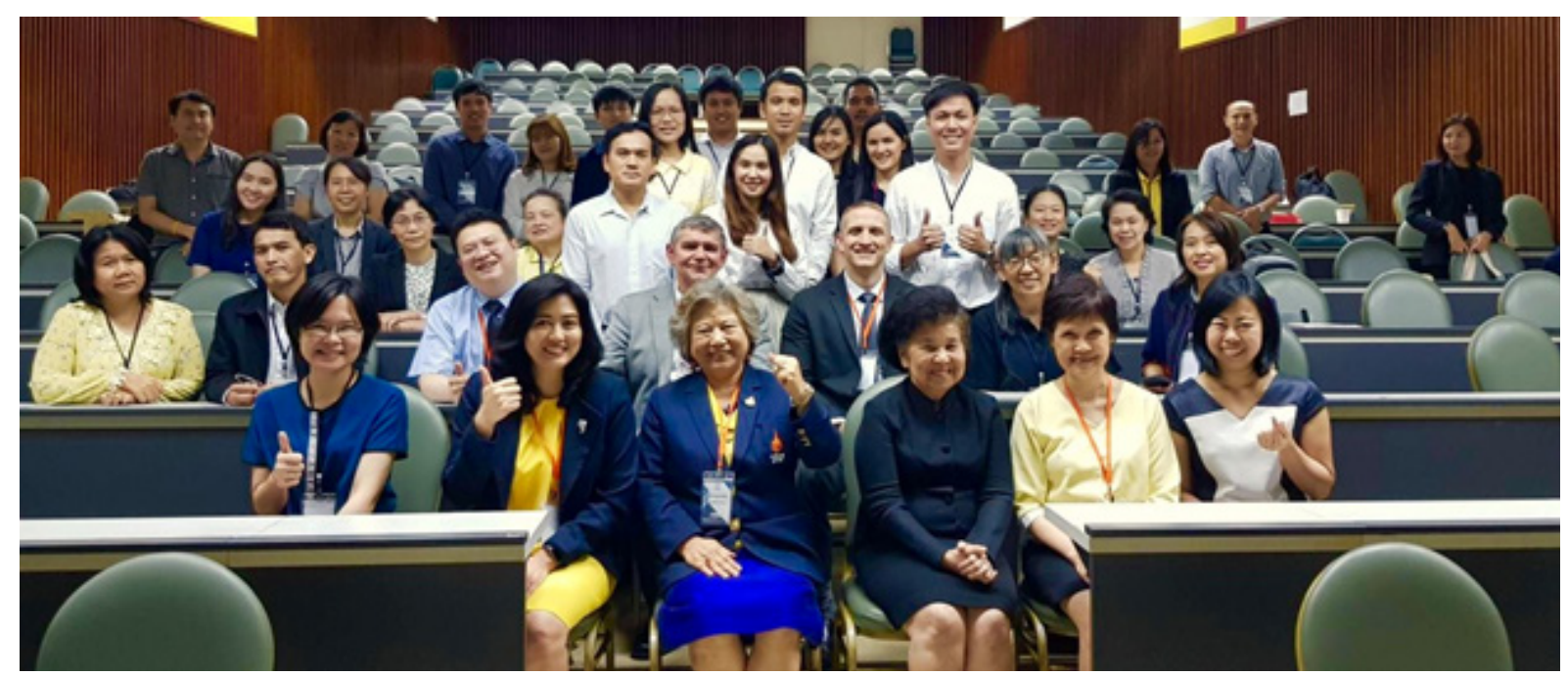

Figure 2. IAEA Expert mission to train local professions on radiation safety and quality practice in diagnostic radiology.

Regarding optimization of medical exposure, setting up the Diagnostic Reference Levels (DRLs) is a tool for optimizing radiation dose which further leads to a safe use of diagnostic radiation. The DRLs project has been carried out by Thailand Ministry of Public Health since 2017 (Figure 3). Currently, DRLs values plain and dental radiography, computed tomography and mammography. The plan is now extended to include Interventional Radiology. National Dose Index Registry starts in the US under the American College of Radiology (ACR). The first high dose modality to be registered is Computed Tomography (CT). Thailand under the IAEA project received the permission to start the first phase of the DIR in 2020. The main purposes of the DIR are to optimize the patient dose and to set up national DRLs on the modality. 


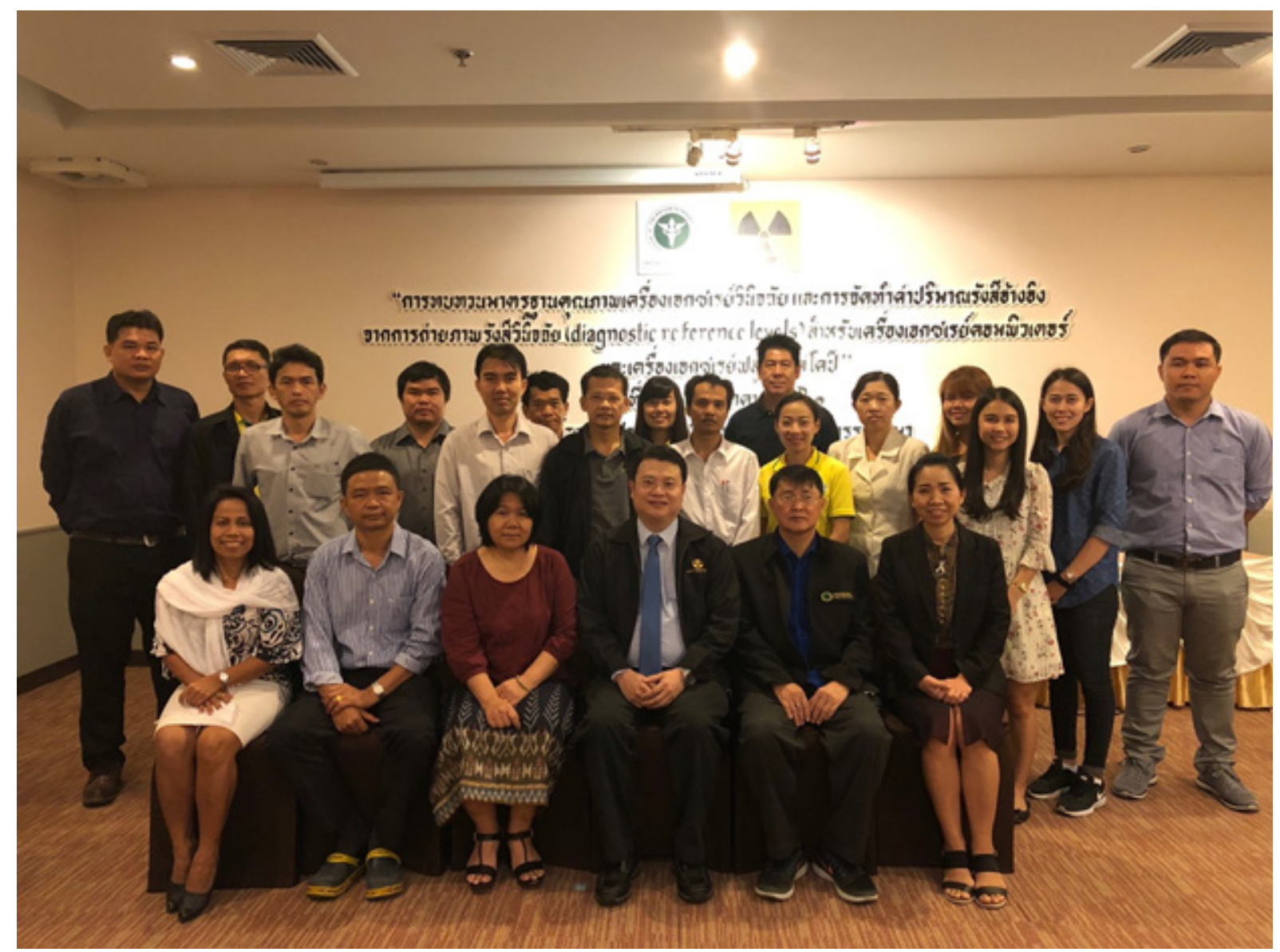

Figure 3. Training on data collection and establishment of the Diagnostic Reference Levels (DRLs) for physicists from the Ministry of Public Health.

In summary, quality and safety in diagnostic radiological services are key elements utilized by the practicioners to ensure the best benefits to patients. We have a future plan to establish Thailand's clinical audit system for radiological services with collaboration among professional bodies (radiologists, medical physicists and radiological technologists). The Diagnostic Reference Levels (DRLs) will be updated every 5 years. The patient dose audit system will also be established. Our experience showed that multi-professional collaboration is a key for success in patient safety and quality in diagnostic radiology. 